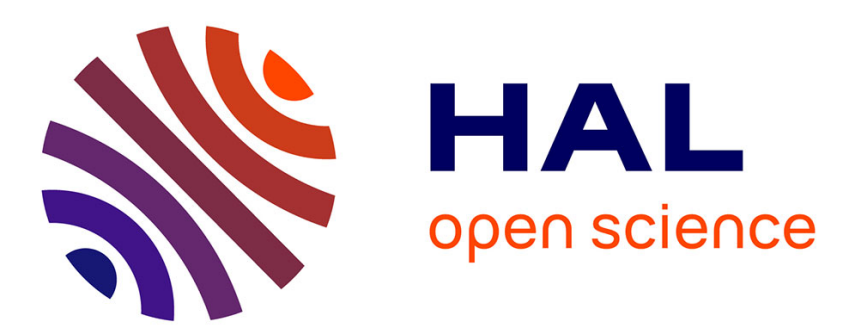

\title{
A new type of impulsive observer for hyperchaotic system
}

Yassine Khaled, Jean-Pierre Barbot, Djamila Benmerzouk, Krishna Busawon

\section{To cite this version:}

Yassine Khaled, Jean-Pierre Barbot, Djamila Benmerzouk, Krishna Busawon. A new type of impulsive observer for hyperchaotic system. IFAC Conference on analysis and control of chaotic systems, Jun 2012, Cancún, México, Mexico. hal-00749718

\section{HAL Id: hal-00749718 \\ https://hal.inria.fr/hal-00749718}

Submitted on 8 Nov 2012

HAL is a multi-disciplinary open access archive for the deposit and dissemination of scientific research documents, whether they are published or not. The documents may come from teaching and research institutions in France or abroad, or from public or private research centers.
L'archive ouverte pluridisciplinaire HAL, est destinée au dépôt et à la diffusion de documents scientifiques de niveau recherche, publiés ou non, émanant des établissements d'enseignement et de recherche français ou étrangers, des laboratoires publics ou privés. 


\title{
A new type of impulsive observer for hyperchaotic system
}

\author{
Y. Khaled ${ }^{*}$ J-P. Barbot ${ }^{* *}$ D. Benmerzouk ${ }^{* * *}$ K. Busawon ${ }^{* * * *}$ \\ * ECS-Lab ENSEA, Cergy-Pontoise, 95014, France. \\ Department of Mathematics, Tlemcen University, Algeria. \\ ** ECS-Lab, ENSEA , Cergy-Pontoise, 95014, France. \\ EPI Non-A, INRIA \\ *** Department of Mathematics, Tlemcen University, Algeria \\ **** School of Computing, Engineering and Information Sciences, \\ Northumbria University, Newcastle upon Tyne NE1 8ST, U.K
}

\begin{abstract}
This paper proposes a new observer scheme for chaotic and hyperchaotic systems. Firstly, a classical impulsive observer is investigated for Lorenz chaotic system. This approach is based on sufficient conditions for stability of impulsive dynamical systems. After, an hybrid observer is proposed for hypoerchaotic systems. Simulation results highlight the well founded of such observer design and show that the discrete measurement may be eventually sparse.
\end{abstract}

Keywords: Impulsive observer, Sliding mode observer, synchronization under sampling, Lorenz system.

\section{INTRODUCTION}

A chaotic dynamical system is a system that is highly sensitive to changes in initial conditions (two very close initial conditions lead to two trajectories that depart quickly from each other) and that evolves in a bounded region in which it has a strange attractor.

The Lyapunov exponent is used to measure the stability degree of this system. A positive Lyapunov exponent (respectively negative) in a direction indicating that a difference between two neighboring trajectories increases (respectively decreases) exponentially with time. When the system has two positive Lyapunov exponents it is called hyperechaotic.

Chaos synchronization (Pecora and Carroll [1990]) attracted a great deal of attention due to its potential applications in many areas such as laser physics, secure communication, chemical reactions, biological systems, etc. Many efficient schemes and techniques have been proposed to synchronize a chaotic systems, such as impulsive control method (Yang and Chua [1997]), adaptive control method (Liao and Tsai [2000]), nonlinear feedback method Chen and Han [2003], sliding mode control (Perruquetti and Barbot [2005]), backstepping design method (Yassen [2006]), etc.

Among these different synchronization techniques, impulsive synchronization showed great potential in chaos communication applications since it uses small impulses generated by samples of the output measurements. It offers a direct method for modulating digital information onto a chaotic carrier signal for the spread spectrum application, also, since these impulses are at discrete times, the redundancy of the synchronization information in the channel will be reduced and therefore the security of the chaos communication system will increase. Moreover, this method is also suitable to deal with systems that cannot endure continuous disturbance. Furthermore, experimental results show that the accuracy of impulsively synchronization depends on both the period and the width of the impulse. Recently, the impulsive synchronization has been pervasively investigated in the literature. For instance in (Itoh et al. [2001]), the authors present conditions under which chaotic systems can be synchronized by impulses determined from samples of their state variables. The authors in (Yang and Chua [1997]) and (Lu and Hill [2007]) propose some sufficient conditions for the impulsive synchronization of chaotic by using the results of (Lakshmikantham et al. [1989]) and linear matrix inequality proprieties (Khadra et al. [2009]). However, these approaches require some restrictive conditions. In addition, it is not easy to find a comparison systems ensuring the stability of the synchronization in some cases.

In this paper, motivated by the above comments, we further investigate a new scheme of synchronization where a single discrete output corresponding to a direction with the positive Lyapunov exponent is received. To solve this problem impulsive dynamical system with small impulses times and sliding mode observer has been used. The latter is used because of its finite time convergence and robustness relative to disturbances. A generalized chaotic Lorenz and hyperchaotic generalized Lorenz systems are taken as an examples to show the results.

The paper is organized as follows. In Section $I I$ a few recalls on impulsive and sliding mode observers are presented. In Section $I I I$, firstly, a new sufficient condition for asymptotic synchronization of chaotic generalized Lorenz system is derived using a discrete Lyapunov function at impulses moments, after a new type of observer is synthesized for generalized hypechaotic generalized Lorenz system. For illustration of the effectiveness of our results, a numerical simulations are given in Section $I V$. 


\section{RECALLS ON IMPULSIVE AND SECOND ORDER SLIDING MODE OBSERVER}

In this section, we recall some basic concepts on impulsive and sliding mode observers which will be used in this paper, for more detail see (Khadra et al. [2009]) and (Barbot et al. [1996]).

\subsection{Impulsive observer}

Consider the nonlinear system with discrete linear outputs:

$$
\left\{\begin{array}{l}
\dot{x}(t)=f(x(t)) \\
y\left(t_{k}\right)=C x\left(t_{k}\right)
\end{array}\right.
$$

where $t_{k} \in T=\left\{t_{i}: i \in \mathbf{N}\right\} \subset \mathbf{R}$ with $t_{i}<t_{i+1}$ for all $i \in \mathbf{N}, x(t) \in \mathbf{R}^{n}$ and $y\left(t_{k}\right) \in \mathbf{R}^{p}$ are the state vector and the discrete output measurement, respectively, $f \in \mathbf{C}^{j}\left(\mathbf{R} \times \mathbf{R}^{n}, \mathbf{R}^{n}\right)^{1}, j \geq 2$ and $C \in \mathbf{R}^{p \times n}$ is a constant matrix.

A classical impulsive observer for system (1) takes the following form:

$$
\left\{\begin{array}{l}
\dot{\hat{x}}(t)=f(\hat{x}(t)) \\
\hat{x}\left(t_{k}^{+}\right)=R \hat{x}\left(t_{k}^{-}\right)+W C x\left(t_{k}^{-}\right)
\end{array}\right.
$$

with $R=I_{n}-W C$, where $I_{n}$ is the identity matrix of dimension $n, t_{k}$ denotes the measurement instant, $t_{k}^{+}$ corresponds to the time just after the $k^{\text {th }}$ measurement and $t_{k}^{-}$is the time just before.

The classical impulsive observer (2) is just a copy of system (1) with a resetting algebraic equation.

Defining the observation error as $e(t)=x(t)-\hat{x}(t)$, the impulsive dynamic of the observation error becomes:

$$
\left\{\begin{array}{l}
\dot{e}(t)=f(x(t))-f(x(t)-e(t)) \\
e\left(t_{k}^{+}\right)=\operatorname{Re}\left(t_{k}\right)
\end{array}\right.
$$

The problem consists to exhibit some sufficient conditions on the impulse gain $R$ and the impulse distance (dwell times) $\theta_{k}=t_{k+1}-t_{k}$ such that the observation error is asymptotically stable.

\subsection{A finite time step-by-step sliding mode observer}

A robust and finite time exact differentiator based on the super twisting algorithm (a second order sliding mode algorithm was introduced by (Levant [1998]) and since successfully applied in many applications. This approach was extended to the design of arbitrary order robust exact differentiators with finite time convergence in (Levant [2005]) using homogeneity properties. In (Barbot et al. [1996]), a so-called step-by-step first order sliding mode observer for the finite time estimation of the state variables was developed. Hereafter, a similar observer based on the super twisting algorithm is given.

Let consider the following system given in the triangular input observer normal form:

${ }^{1} \mathbf{C}^{j}$ is the set of functions of class $j$ defined from $\mathbf{R} \times \mathbf{R}^{n} \rightarrow \mathbf{R}^{n}$.

$$
\begin{cases}\dot{x}_{1} & =x_{2} \\ \dot{x}_{2} & =x_{3} \\ \vdots & \\ \dot{x}_{n-1} & =x_{n} \\ \dot{x}_{n} & =g\left(x_{1}, \ldots, x_{n-1}, x_{n}\right) \\ y & =x_{1}\end{cases}
$$

The Super Twisting Algorithm is given by the following structure:

$$
\sum_{o b s}=\left\{\begin{array}{l}
u\left(e_{1}\right)=u_{1}+\lambda_{1}\left|e_{1}\right|^{\frac{1}{2}} \operatorname{sign}\left(e_{1}\right) \\
\dot{u}_{1}=\alpha_{1} \operatorname{sign}\left(e_{1}\right)
\end{array}\right.
$$

where $e_{1}=x_{1}-\hat{x}_{1}$ and $\lambda_{1}, \alpha_{1}$ are positive parameters and $u_{1}$ is the differentiator output.

The step by step exact differentiator applied to (4) leads to the following form:

$$
\left\{\begin{array}{l}
\dot{\hat{x}}_{1}=\tilde{x}_{2}+\lambda_{1}\left|x_{1}-\hat{x}_{1}\right|^{\frac{1}{2}} \operatorname{sign}\left(x_{1}-\hat{x}_{1}\right) \\
\dot{\tilde{x}}_{2}=\alpha_{1} \operatorname{sign}\left(x_{1}-\hat{x}_{1}\right) \\
\dot{\hat{x}}_{2}=E_{1}\left[\tilde{x}_{3}+\lambda_{2}\left|\tilde{x}_{2}-\hat{x}_{2}\right|^{\frac{1}{2}} \operatorname{sign}\left(\tilde{x}_{2}-\hat{x}_{2}\right)\right] \\
\vdots \\
\dot{\tilde{x}}_{n}=E_{n-2} \alpha_{n-1} \operatorname{sign}\left(\tilde{x}_{n-1}-\hat{x}_{n-1}\right) \\
\dot{\hat{x}}_{n}=E_{n-1}\left[\tilde{\theta}+\lambda_{n}\left|\tilde{x}_{n}-\hat{x}_{n}\right|^{\frac{1}{2}} \operatorname{sign}\left(\tilde{x}_{n}-\hat{x}_{n}\right)\right] \\
\dot{\tilde{\theta}}=E_{n-1} \alpha_{n} \operatorname{sign}\left(\tilde{x}_{n}-\hat{x}_{n}\right)
\end{array}\right.
$$

with $\tilde{x}_{1}=x_{1}$ and $[\tilde{x}, \tilde{\theta}]^{T}=\left[\tilde{x}_{1}, \tilde{x}_{2}, \ldots, \tilde{x}_{n}, \tilde{\theta}\right]^{T}$ is the output of the observer. For $i=1, \ldots, n-1$, the scalar functions $E_{i}$ are defined as: $E_{i}=1$ if $\left|\tilde{x}_{j}-\hat{x}_{j}\right|<\epsilon$, for all $j \leq i$ else $E_{i}=0, \epsilon$ is a small positive constant. The observer gains $\lambda_{i}$ and $\alpha_{i}$ are positive scalars.

The convergence of the state observation error is obtained in $(n-1)$ steps and in finite time. Moreover, this observer is robust against noise and some disturbances.

Applying the exact differentiator (5) to system (4) when $n=2$, one has only one step to do and obtains:

$$
\left\{\begin{array}{l}
\dot{\hat{x}}_{1}=\hat{x}_{2}+\lambda_{1}\left|x_{1}-\hat{x}_{1}\right|^{\frac{1}{2}} \operatorname{sign}\left(x_{1}-\hat{x}_{1}\right) \\
\dot{\hat{x}}_{2}=g\left(x_{1}, \tilde{x}_{2}\right)+\alpha_{1} \operatorname{sign}\left(x_{1}-\hat{x}_{1}\right)
\end{array}\right.
$$

Theorem 1. (Davila et al. [2005]) Consider the system (4) with $n=2$, assumed to be defined by bounded state in finite time, and the observer based on the differentiator (7). For any initial conditions $x(0), \hat{x}(0)$, there exists a choice of $\lambda_{1}$ and $\alpha_{1}$ such that the observer state $\hat{x}$ converges in finite time to $x$, i.e. $\left(\hat{x}_{1} ; \hat{x}_{2}\right) \rightarrow\left(x_{1} ; x_{2}\right)$.

Remark 1. These observers, because of their relative robustness proprite may, in addition, be applied to systems subjected to discontinuites, disturbances or parameter uncertainties.

\section{TWO TYPES OF OBSERVER DESIGN}

In this section, two hybrid observers are proposed that can estimate the states of chaotic systems using only a single discrete output. First, we consider chaotic systems with only one positive Lyapunov exponent. Next, we extent our study to the Hyperchaotic systems (more than one positive Lyapunov exponent). 


\subsection{Observer design for Lorenz chaotic system:}

Lorenz chaotic system was studied by many authors, particulary for characterizing chaotic chemical reactions. The nonlinear differential Lorenz system is described by:

$$
\left\{\begin{array}{l}
\dot{x}_{1}=a\left(x_{3}-x_{1}\right) \\
\dot{x}_{2}=-b x_{2}+x_{1} x_{3} \\
\dot{x}_{3}=-c x_{1}+d x_{3}-x_{1} x_{2} \\
y\left(t_{k}\right)=x_{3}\left(t_{k}\right)
\end{array}\right.
$$

where $x_{1}(t), x_{2}(t)$ and $x_{3}(t)$ are the state variables, and $a=35, b=3, c=7$ and $d=12$

Remark 2. Regardless to their initial conditions, chaotic systems have bounded states so that one can find a positive number $M$ such that $\sup _{t}\left|x_{i}(t)\right| \leq M, \forall i=1,2,3$, for any initial conditions on the strong attractor.

The proposed impulsive observer corresponding to system (8) is designed as follows:

$$
\begin{cases}\dot{\hat{x}}_{1} & =a\left(\hat{x}_{3}-\hat{x}_{1}\right) \\ \dot{\hat{x}}_{2} & =-b \hat{x}_{2}+\hat{x}_{1} \hat{x}_{3} \\ \dot{\hat{x}}_{3} & =-c \hat{x}_{1}+d \hat{x}_{3}-\hat{x}_{1} \hat{x}_{2} \\ \hat{x}_{3}\left(t_{k}^{+}\right) & =r \hat{x}_{3}\left(t_{k}\right)+(1-r) x_{3}\left(t_{k}\right)\end{cases}
$$

Where $r$ is a fixed real number. Moreover, in order to ensure the observer state, bondless saturations are added on $\hat{x}_{1}, \hat{x}_{2}$ and $\hat{x}_{3}$.

Note that, the system (9) has a same continuous dynamics than the system (8), except that at each sampling time the observer state jumps proportionally to the error between the estimate output $\hat{y}\left(t_{k}\right)$ and the measured state $y\left(t_{k}\right)$.

Theorem 2. If $|r|<\frac{1}{\sqrt{1+2 d \theta_{k}}}$, then, there exists $\theta^{\text {max }}$ such that, for any $k$ verifying $t_{k+1}-t_{k} \leq \theta^{\max }$, the states of the observer (9) converge practically ${ }^{2}$ to the states of the system (8).

Remark 1. It is important to note that the output is imposed in this example. Nevertheless, if the choice of the output is free, the output can be determined according to observability coefficients see Letellier et al. [2005].

Proof. The observation error $e_{i}=x_{i}-\hat{x}_{i}$ are described by

$$
\begin{cases}\dot{e}_{1} & =a\left(e_{3}-e_{1}\right) \\ \dot{e}_{2} & =-b e_{2}+x_{1} x_{3}-\hat{x}_{1} \hat{x}_{3} \\ \dot{e}_{3} & =-c e_{1}+d e_{3}-x_{1} x_{2}+\hat{x}_{1} \hat{x}_{2} \\ e_{3}\left(t_{k}^{+}\right) & =r e_{3}\left(t_{k}\right)\end{cases}
$$

first we decompose the system into two subsystems $z_{1}=\left(e_{1}, e_{2}\right)^{T}$ and $z_{2}=e_{3}$.

Define the Lyapunov function as:

$$
V(z)=V_{1}\left(z_{1}\right)+V_{2}\left(z_{2}\right)
$$

with $V_{1}\left(z_{1}\left(t_{k}\right)\right)=z_{1}\left(t_{k}\right)^{T} \alpha z_{1}\left(t_{k}\right), V_{2}\left(z_{2}\left(t_{k}\right)\right)=\beta z_{2}^{2}\left(t_{k}\right)$ and $\alpha=\operatorname{diag}\left\{\alpha_{1}, \alpha_{2}\right\}, \alpha_{i}>0$ and $\beta$ a real positive number.

Define the Lyapunov difference at the impulse time $t_{k}$ as:

$$
\begin{aligned}
\triangle V\left(z\left(t_{k}\right)\right) & =V\left(z\left(t_{k+1}^{+}\right)\right)-V\left(z\left(t_{k}^{+}\right)\right) \\
& =\triangle V_{1}\left(z_{1}\left(t_{k}\right)\right)+\triangle V_{2}\left(z_{2}\left(t_{k}\right)\right)
\end{aligned}
$$

2 Is means that the observation error $e(t)$ converges to a ball $B(0, \epsilon)$
Now, since $e_{1}\left(t_{k}^{+}\right)=e_{1}\left(t_{k}\right)$ and $e_{2}\left(t_{k}^{+}\right)=e_{2}\left(t_{k}\right)$, the first difference is:

$$
\begin{aligned}
\triangle V_{1}\left(z_{1}\left(t_{k}\right)\right) & =z_{1}^{T}\left(t_{k+1}\right) \alpha z_{1}\left(t_{k+1}\right)-z_{1}^{T}\left(t_{k}\right) \alpha z_{1}\left(t_{k}\right) \\
& =\alpha_{1} e_{1}^{2}\left(t_{k+1}\right)+\alpha_{2} e_{2}^{2}\left(t_{k+1}\right) \\
& -\alpha_{1} e_{1}^{2}\left(t_{k}\right)-\alpha_{2} e_{2}^{2}\left(t_{k}\right)
\end{aligned}
$$

Moreover, from the Taylor development of $e_{i}(t)$ at $\theta_{k}=t_{k+1}-t_{k}$ (which is very small), one has:

$$
e_{i}\left(t_{k+1}\right)=e_{i}\left(t_{k}^{+}\right)+\left.\theta_{k} \frac{d e_{i}}{d t}\right|_{t=t_{k}^{+}}+o\left(\theta_{k}^{2}\right)
$$

for the sake of simplicity, setting $e_{i}\left(t_{k}^{+}\right)=e_{i}$, and as $\theta_{k}$ is small enough, then all terms of the form $\gamma(t) o\left(\theta_{k}^{2}\right)$ are negligible for any $\gamma($.$) bounded.$

Therefore

$$
\begin{aligned}
\triangle V_{1}\left(z_{1}\left(t_{k}\right)\right) & =\alpha_{1}\left[e_{1}+\theta_{k}\left(-a e_{1}+a e_{3}\right)+o\left(\theta_{k}^{2}\right)\right]^{2} \\
& +\alpha_{2}\left[e_{2}+\theta_{k}\left(-b e_{2}+x_{1} x_{3}-\hat{x}_{1} \hat{x}_{3}\right)+o\left(\theta_{k}^{2}\right)\right]^{2} \\
& -\alpha_{1} e_{1}^{2}-\alpha_{2} e_{2}^{2} \\
& =\alpha_{1}\left[e_{1}^{2}+2 e_{1} \theta_{k}\left(-a e_{1}+a e_{3}\right)+o\left(\theta_{k}^{2}\right)\right] \\
& +\alpha_{2}\left[e_{2}^{2}+2 e_{2} \theta_{k}\left(-b e_{2}+x_{1} x_{3}-\hat{x}_{1} \hat{x}_{3}\right)+o\left(\theta_{k}^{2}\right)\right] \\
& -\alpha_{1} e_{1}^{2}-\alpha_{2} e_{2}^{2}
\end{aligned}
$$

Since $x_{1} x_{3}-\hat{x}_{1} \hat{x}_{3}=x_{1} e_{3}+x_{3} e_{1}-e_{1} e_{3}$, we obtain:

$$
\begin{aligned}
\triangle V_{1}\left(z_{1}\left(t_{k}\right)\right) & =2 \alpha_{1} \theta_{k} e_{1}\left(-a e_{1}+a e_{3}\right) \\
& +2 \alpha_{2} \theta_{k} e_{2}\left(-b e_{2}+x_{1} e_{3}+x_{3} e_{1}-e_{1} e_{3}\right) \\
& +o\left(\theta_{k}^{2}\right)
\end{aligned}
$$

similarly, for the second difference we obtain:

$$
\begin{aligned}
\triangle V_{2}\left(z_{2}\left(t_{k}\right)\right) & =V_{2}\left(z_{2}\left(t_{k+1}^{+}\right)\right)-V_{2}\left(z_{2}\left(t_{k}^{+}\right)\right) \\
& =V_{2}\left(r z_{2}\left(t_{k+1}\right)\right)-V_{2}\left(z_{2}\left(t_{k}^{+}\right)\right) \\
& =r^{2} \beta\left[e_{3}+\theta_{k}\left(-c e_{1}+d e_{3}-x_{1} x_{2}+\hat{x}_{1} \hat{x}_{2}\right)\right. \\
& \left.+o\left(\theta_{k}^{2}\right)\right]^{2}-\beta e_{3}^{2} \\
\triangle V_{2}\left(z_{2}\left(t_{k}\right)\right) & =\beta\left(r^{2}+2 d r^{2} \theta_{k}-1\right) e_{3}^{2} \\
& +2 r^{2} \beta \theta_{k} e_{3}\left(-c e_{1}-x_{1} e_{2}-x_{2} e_{1}+e_{1} e_{2}\right) \\
& +o\left(\theta_{k}^{2}\right)
\end{aligned}
$$

The convergence of the state observation error is obtained in three steps.

First step:

According to the hypothesis of the theorem, we have $|r|<\frac{1}{\sqrt{1+2 d \theta_{k}}}$, then $\beta\left(r^{2}+2 d r^{2} \theta_{k}-1\right) e_{3}^{2}<0$ and with respect to the fact that $\sup _{t}\left|x_{i}\right|<M$ and $\sup _{t}\left|\hat{x}_{i}\right|<M$, then

$$
\begin{aligned}
\triangle V_{2}\left(z_{2}\left(t_{k}\right)\right) & \leq \beta\left(r^{2}+2 d r^{2} \theta_{k}-1\right) e_{3}^{2} \\
& +2 r^{2} \beta \theta_{k}\left|e_{3}\right|\left(c M+8 M^{2}\right) \\
& +o\left(\theta_{k}^{2}\right)
\end{aligned}
$$

So, there exists $e_{3}^{\prime}=\left|\frac{2 r^{2} \theta_{k} M(c+8 M)}{r^{2}\left(1+2 d \theta_{k}\right)-1}\right|$, such that

$$
\forall\left|e_{3}\right|>e_{3}^{\prime}: \triangle V_{2}<0
$$


and consequently for all $\varepsilon>0$ there exists $k^{\prime}$, such that

$$
\forall k>k^{\prime}: e_{3}\left(t_{k}\right)<e_{3}^{\prime}+\varepsilon
$$

\section{Second step:}

Using (13) in (11), we obtain:

$$
\begin{aligned}
\triangle V_{1}\left(z_{1}\left(t_{k}\right)\right) & \leq 2 \alpha_{1} \theta_{k} e_{1}\left(-a e_{1}+a\left(e_{3}^{\prime}+\varepsilon\right)\right) \\
& +2 \alpha_{2} \theta_{k} e_{2}\left(-b e_{2}+x_{1}\left(e_{3}^{\prime}+\varepsilon\right)\right. \\
& \left.+x_{3} e_{1}+e_{1}\left(e_{3}^{\prime}+\varepsilon\right)\right)+o\left(\theta_{k}^{2}\right)
\end{aligned}
$$

with $e_{3}^{\prime}=\left|\frac{2 r^{2} M(c+8)}{r^{2}\left(1+d \theta_{k}\right)-1}\right| \theta_{k}$, then we get:

$$
\begin{aligned}
\triangle V_{1}\left(z_{1}\left(t_{k}\right)\right) & \leq \theta_{k}\left(-2 \alpha_{1} a e_{1}^{2}-2 \alpha_{2} b e_{2}^{2}+2 \alpha_{2} x_{3} e_{1} e_{2}\right)+o\left(\theta_{k}^{2}\right) \\
& \leq \theta_{k}\left(-2 \alpha_{1} a e_{1}^{2}-2 \alpha_{2} b e_{2}^{2}+2 \alpha_{2} M e_{1} e_{2}\right)+o\left(\theta_{k}^{2}\right) \\
& =-\theta_{k}\left(\sqrt{2 \alpha_{1} a} e_{1}-\frac{\alpha_{2} M}{\sqrt{\alpha_{1} a}} e_{2}\right)^{2} \\
& -\theta_{k}\left(2 \alpha_{2} b-\frac{\alpha_{2}^{2} M^{2}}{\alpha_{1} a}\right) e_{2}^{2}+o\left(\theta_{k}^{2}\right)
\end{aligned}
$$

Since $\alpha_{1}$ and $\alpha_{2}$ are chosen arbitrarily, for every fixed $\epsilon_{1}$ and $\epsilon_{2}$, for all $\left|e_{1}\right|>\epsilon_{1}$ and $\left|e_{2}\right|>\epsilon_{2}$, we have

$$
\triangle V_{1}\left(z_{1}\left(t_{k}\right)\right)<0
$$

\section{Third step:}

Finally, using the fact that $\left|e_{1}\right|>\epsilon_{1}$ and $\left|e_{2}\right|>\epsilon_{2}$, the inequality (12) becomes

$$
\begin{aligned}
\triangle V_{2}\left(z_{2}\left(t_{k}\right)\right) & \leq \beta\left(r^{2}+2 d r^{2} \theta_{k}-1\right) e_{3}^{2} \\
& +2 r^{2} \beta \theta_{k} e_{3}\left(c \epsilon_{1}+M \epsilon_{1}+M \epsilon_{2}+\epsilon_{1} \epsilon_{2}\right)(16) \\
& +o\left(\theta_{k}^{2}\right)
\end{aligned}
$$

which implies that for $\theta_{k}<\theta^{\max }$, where $\theta^{\max }$ is the smallest value of $\theta_{k}$ ensuring (13) and (14) and in any case that the terms in $o\left(\theta_{k}^{2}\right)$ are negligible, and from (15) it is possible to set $\epsilon_{3}=\left|\frac{2 r^{2} \theta_{k}\left((c+M) \epsilon_{1}+M \epsilon_{2}+\epsilon_{1} \epsilon_{2}\right)}{r^{2}\left(1+2 d \theta_{k}\right)-1}\right|$, such that for all $\left|e_{3}\right|>\epsilon_{3}$, we have:

$$
\triangle V_{2}\left(z_{2}\left(t_{k}\right)\right)<0
$$

Those with respect to (14) and (17), the observation error (10) is practically stable to a ball of radius $\epsilon=\left(\epsilon_{1}, \epsilon_{2}, \epsilon_{3}\right)^{T}$.

In the case $r=0$, we obtain $e_{3}^{\prime}=0$, then the practical stability of (10) is obtained in one step because all the term in $e_{3}$ will be canceled, and so the stability analyzing is reduced to the second step of the above proof.

Unlike the linear case, we can not involve an LMI (Linear Matrix Inequality) in the proof because of the quadratic term in (8), which generate a cubic term $\left(e_{1}, e_{2}, e_{3}\right)$ in $\triangle V$.

Remark 3. The theorem 2 means that the number of outputs must be at least equal to the number of positive Lyapunov exponents, for such kind of observer. This seems to be in accordance with Pyragas conjecture (Pyragas give is conjecture for continuous measurements (Pyragas [1993])) but was contradicted in (Itoh et al. [2001]) and (Boutat-Baddas et al. [2009]). However, applied to impulsive observer, it is fully expected that the conjecture is true in this particular case.
It is important to note that the observability or detectability conditions are not sufficient for this kind of observer design, because the measurements are discrete. It is necessary to add a condition for unstable states, here it is assumed that they are measured. In the next section, we relax this condition and it is also shown that the Pyragas conjecture is also refuted for impulsive synchronization.

\subsection{Observer for hyperchaotic Lorenz system}

In the case of hyperchaotic system the number of positive Lyapunov exponents is more than one, the generalized Lorenz hyperchaotic system is described by

$$
\left\{\begin{array}{l}
\dot{x}_{1}=a\left(x_{3}-x_{1}\right) \\
\dot{x}_{2}=-b x_{2}+x_{1} x_{3} \\
\dot{x}_{3}=-c x_{1}+d x_{3}-x_{1} x_{2}+x_{4} \\
\dot{x}_{4}=-k x_{1}+j x_{4} \\
y\left(t_{k}\right)=x_{3}\left(t_{k}\right)
\end{array}\right.
$$

with: $a=35, b=3, c=7, d=12, k=5$ and $j=0.5$.

First, it is important to note that if a classical impulsive observer design is applied to this kind of systems, the observer will diverge, because only one unstable direction is measured $x_{3}\left(t_{k}\right)$. However, the unmeasured dynamic $x_{4}(t)$ corresponding to the second positive Lyapunov exponent causes the divergence of the observer. For this reason, if an impulsive observer is considered, it is judicious to add an auxiliary observer in order to guarantee the convergence of observer see (Fig. 1).

Similar, to the previously presented observer, an impulsive observer coupled with a super twisting continuous observer is proposed. This latter is used to reconstruct the other unstable states from the impulsive output.

The complete proposed impulsive generalized observer corresponding to system (18) is designed as follows:

$$
\begin{cases}\dot{\hat{x}}_{1} & =a\left(\hat{x}_{3}-\hat{x}_{1}\right) \\ \dot{\hat{x}}_{2} & =-b \hat{x}_{2}+\hat{x}_{1} \hat{x}_{3} \\ \dot{\hat{x}}_{3} & =c \hat{x}_{1}+d \hat{x}_{3}-\hat{x}_{1} \hat{x}_{2}+\hat{x}_{4} \\ \hat{\hat{x}}_{4} & =-k \hat{x}_{1}+j \hat{x}_{4}+r_{1}\left(z_{4}-\hat{x}_{4}\right) \\ \hat{x}_{3}\left(t_{k}^{+}\right) & =r_{2} \hat{x}_{3}\left(t_{k}\right)+\left(1-r_{2}\right) x_{3}\left(t_{k}\right)\end{cases}
$$

and

$$
\left\{\begin{array}{l}
\dot{z}_{3}=z_{d 3}+\lambda_{1}\left|z_{3}-\hat{x}_{3}\right|^{\frac{1}{2}} \operatorname{sign}\left(z_{3}-\hat{x}_{3}\right) \\
\dot{z}_{d 3}=\alpha_{1} \operatorname{sign}\left(z_{3}-\hat{x}_{3}\right)
\end{array}\right.
$$

with

$$
z_{4}=z_{d 3}-c \hat{x}_{1}-d z_{3}+\hat{x}_{1} \hat{x}_{2}
$$

where $\lambda_{1}>0, \alpha_{1}>0$ and $\left|r_{2}\right|<1$.

The impulsive observer plays the predictor role for the state $x_{3}$. The continuity of the super twisting observer avoid jumps at $t_{k}$ instants. Moreover, since it is robust to noise and disturbance, the observer does not change abruptly at the impulse time, which gives us a better estimate of $\hat{x}_{3}$ with respect to the impulsive output.

\subsection{Numerical simulation}

In order to demonstrate and verify the performance of the proposed method, some numerical simulations are presented in this section. 


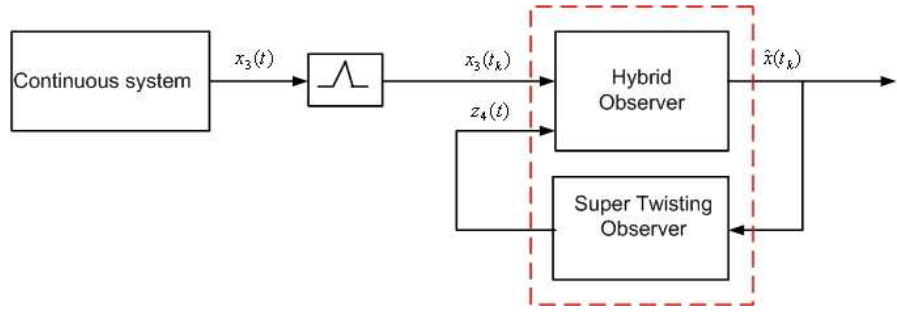

Fig. 1. Impulsive generalized Observer
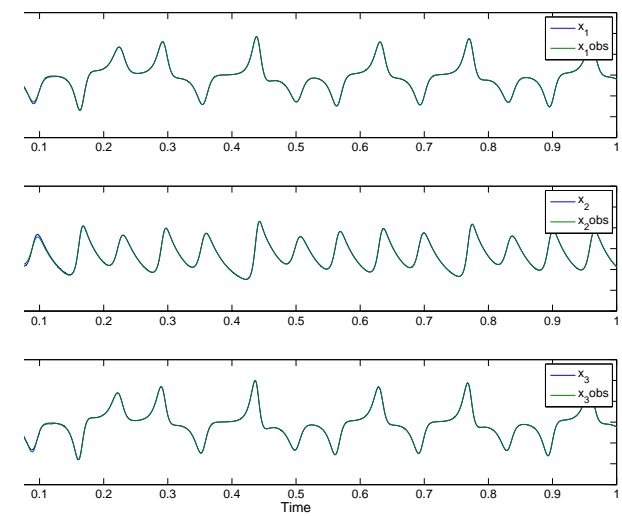

Fig. 2. $x(-)$ and $\hat{x}(-)$
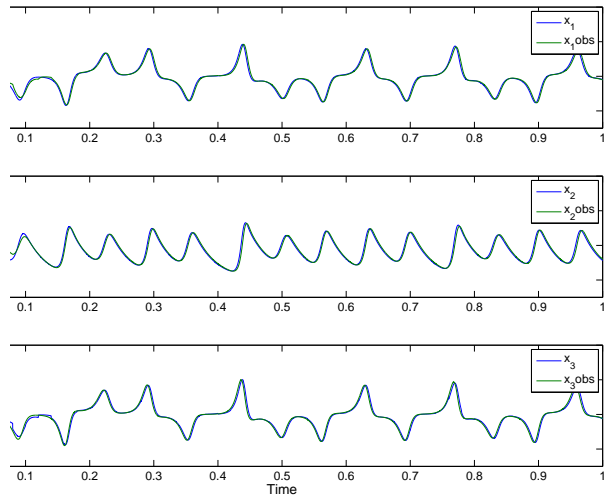

Fig. 3. $x(-)$ and $\hat{x}(-)$

\subsection{Chaotic Lorenz system}

The chaotic generalized Lorenz system is given in (8) with a modified parameters $a=350, b=30, c=70$ and $d=$ 120 , in order to increase the fundamental frequency of system $(f=154 \mathrm{~Hz})$. The solution of this system with initial conditions $x(0)=(5,2,1)^{T}$ is obtained numerically. The initial conditions for the observer $(9)$ is $\hat{x}=(-0.6,-2,0)^{T}$ and impulse gain $r=0$. Fig. 2 show the performance of the observer (9) with impulse distance $\theta_{k} \in[0.02,0.05]$ such that it does not satisfy the Nyquist-Shannon theorem, i.e the sampling frequency of output measures $\left(\frac{1}{\theta_{k}}=50 \mathrm{~Hz}\right)$ is less than the double of fundamental frequency of the chaotic system (8)

Now, starting from the same initial conditions, we add an output noise in order to show the robustness of the observer in this case. Moreover, we have chosen a Butter-

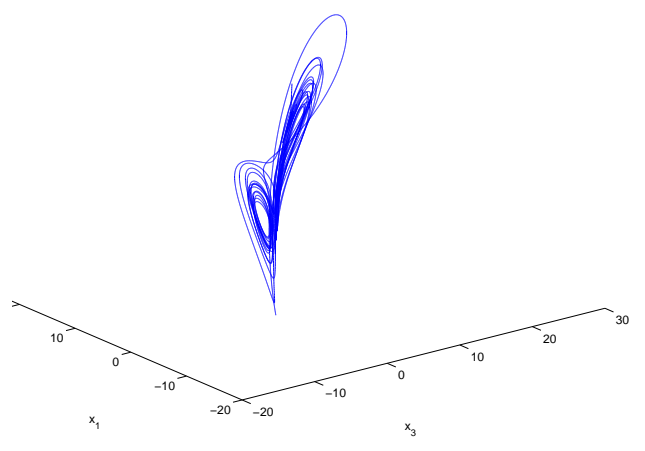

Fig. 4. Phase graph of HyperChaotic generalized Lorenz
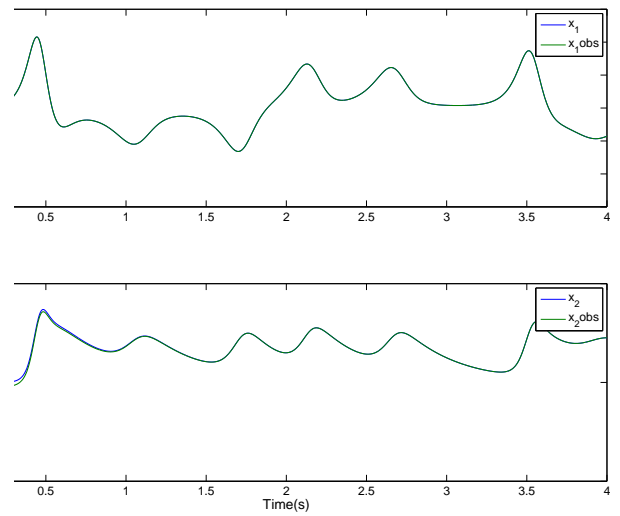

Fig. 5. $x_{1}(-), x_{2}(-)$ and $\hat{x}_{1}(-), \hat{x}_{2}(-)$

worth filter of order three with bandwidth equal to $192 \mathrm{~Hz}$. Fig. 3 show that the observer states follow the system states.

\subsection{Hyperchaotic generalized Lorenz system}

The Hyperchaotic generalized Lorenz system is given in (18) where $a=35, b=3, c=7, d=12$ and $k=5$. Typical phase portrait of this system with initial conditions $x(0)=(0.005,0.01,0.05,0.5)^{T}$ is plot in Fig. 4 .

The observer (19) is designed with the following parameters; impulses times $\theta_{k} \in[0.002,0.005]$, initial conditions $\hat{x}(0)=(-2,-5,0,0.4)$ and gains observability $\hat{x}=(-2,-5,0.4), \lambda_{1}=70, \alpha_{1}=9500, m=5$ and $r=0$.

Fig. 5 and 6 shows the performance of the observer. The peaks in $\hat{x}_{4}$ are due to jumps of $\hat{x_{3}}$ into $x_{3}$ as it is highlighted in Fig. 6. Similarly with the previous example, we add output noise, Fig. 7 and 8 illustrate the performance of proposed observer with a delay of $10^{-2}$ due to the filter except that the observer takes a little time compared to the first case louse converge.

\section{CONCLUSION}

In this paper, we have shown that it is possible to design an observer for chaotic and hyperchaotic Lorenz system using only a single discrete measurement. Two types of 

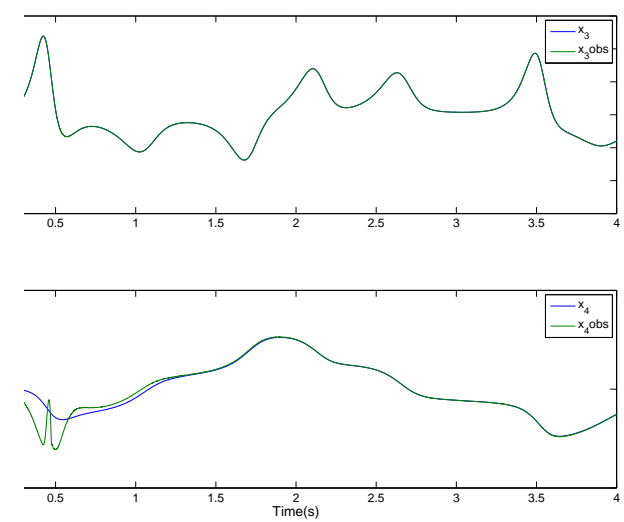

Fig. 6. $x_{3}(-), x_{4}(-)$ and $\hat{x}_{3}(-), \hat{x}_{4}(-)$
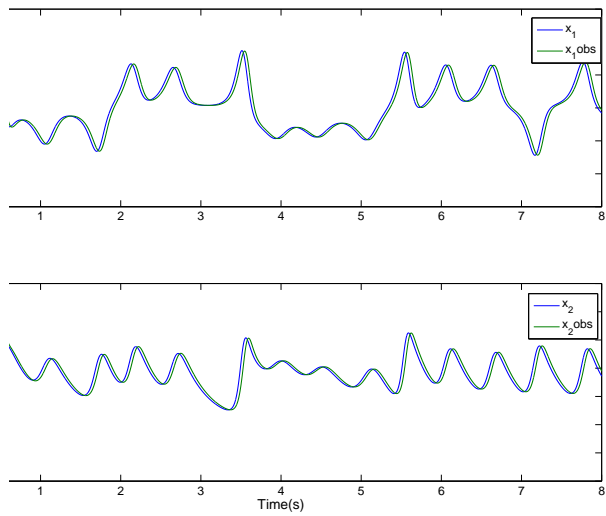

Fig. 7. $x_{1}(-)$ and $\hat{x}_{2}(-)$
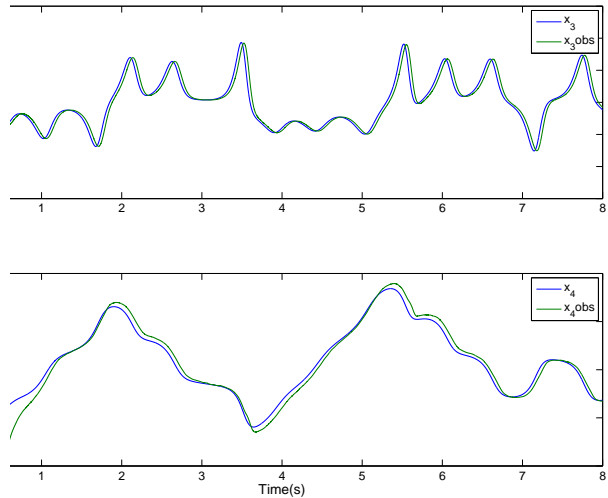

Fig. 8. $x_{3}(-)$ and $\hat{x}_{4}(-)$

observers have been designed, one (impulsive) for the generalized Lorenz chaotic system, and the other (impulsive coupled with super twisting continuous observer) for the generalized Lorenz hyperchaotic system. The design is based on the study of the stability of impulsive dynamical systems. The simulation results obtained confirmed the good performance of the observer.

\section{REFERENCES}

Barbot, J.P., Boukhobza, T., and Djemai, M. (1996). Sliding mode observer for triangular input form. In Decision and Control, 1996., Proceedings of the 35th IEEE, volume 2, 1489-1490. IEEE.

Boutat-Baddas, L., Boutat, D., and Barbot, J.P. (2009). Observability analysis by poincaré normal forms. Mathematics of Control, Signals, and Systems (MCSS), 21(2), 147-170.

Chen, M. and Han, Z. (2003). Controlling and synchronizing chaotic genesio system via nonlinear feedback control. Chaos, Solitons $\&$ Fractals, 17(4), 709-716.

Davila, J., Fridman, L., and Levant, A. (2005). Secondorder sliding-mode observer for mechanical systems. Automatic Control, IEEE Transactions on, 50(11), 17851789.

Itoh, M., Yang, T., and Chua, L.O. (2001). Conditions for impulsive synchronization of chaotic and hyperchaotic systems. International Journal of Bifurcation and Chaos in Applied Sciences and Engineering, 11(2), 551-560.

Khadra, A., Liu, X.Z., and Shen, X. (2009). Analyzing the robustness of impulsive synchronization coupled by linear delayed impulses. Automatic Control, IEEE Transactions on, 54(4), 923-928.

Lakshmikantham, V., Bănov, D., and Simeonov, P.S. (1989). Theory of impulsive differential equations, volume 6. World Scientific Pub Co Inc.

Letellier, C., Aguirre, L., and Maquet, J. (2005). Relation between observability and differential embeddings for nonlinear dynamics. Physical Review E, 71, 066213.

Levant, A. (1998). Robust exact differentiation via sliding mode technique. Automatica, 34(3), 379-384.

Levant, A. (2005). Homogeneity approach to high-order sliding mode design. Automatica, 41(5), 823-830.

Liao, T.L. and Tsai, S.H. (2000). Adaptive synchronization of chaotic systems and its application to secure communications. Chaos, Solitons \& Fractals, 11(9), $1387-1396$.

Lu, J.G. and Hill, D.J. (2007). Impulsive synchronization of chaotic lur'e systems by linear static measurement feedback: An lmi approach. Circuits and Systems II: Express Briefs, IEEE Transactions on, 54(8), 710-714.

Pecora, L.M. and Carroll, T.L. (1990). Synchronization in chaotic systems. Physical review letters, 64(8), 821-824.

Perruquetti, W. and Barbot, J.P. (2005). Chaos in automatic control,(control engineering, vol. 18).

Pyragas, K. (1993). Predictable chaos in slightly perturbed unpredictable chaotic systems. Physics Letters A, 181(3), 203-210.

Yang, T. and Chua, L.O. (1997). Impulsive stabilization for control and synchronization of chaotic systems: Theory and application to secure communication. Circuits and Systems I: Fundamental Theory and Applications, IEEE Transactions on, 44(10), 976-988.

Yassen, M.T. (2006). Chaos control of chaotic dynamical systems using backstepping design. Chaos, Solitons 8 Fractals, 27(2), 537-548. 